\title{
One-step simple assay to determine antigen-specific cytotoxic activities by single-color flow cytometry
}

\author{
Yohko Nakagawa* ${ }^{*}$ Eiji Watari*, Masumi Shimizu, and Hidemi TaKahashi \\ Department of Microbiology and Immunology, Nippon Medical School, Sendagi, Bunkyo-ku Tokyo 113-8602, JAPAN \\ (Received 7 January 2011; and accepted 26 January 2011)
}

\begin{abstract}
Assays for cytotoxicity of CTLs in vivo using a fluorescent-based dye, 5- (and 6-) carboxyfluorescein diacetate succinimydyl ester (CFSE), have been established and widely used. On the basis of this experience, we applied it to in vitro assay system and established a simpe, highly sensitive flow cytometric assay for CTL activity. In our assay, specific activities of CTLs could be detected by a reduction in sensitive target cell numbers on single-color histogram plot analysis. By using this assay, we could determine the changes in cytotoxic activity by single amino acid substitution within an epitope peptide. Adherent cells were also used as target cells in this assay by treatment with excess EDTA and trypsin reagents after incubation with effector CTLs. Furthermore, when fluorescent calibration beads were used as a control, we could determine the cytotoxicity of CTLs against tumor cells. The results obtained from our assay were almost consistent with those from the conventional ${ }^{51} \mathrm{Cr}$-release assay. Because our assay uses only a stable non-radioactive reagent, CFSE, this assay is safe, inexpensive and extremely easy. These results indicated that this new assay (FACS-CTL assay) would be sufficiently acceptable alternative to classical ${ }^{51} \mathrm{Cr}$-release assay.
\end{abstract}

Antigen-specific $\mathrm{CD}^{+}$cytotoxic $\mathrm{T}$ lymphocytes (CTLs) play an important role in preventing both viral infection and disease progression. Therefore, measurement of cytotoxic activities of these CTLs is needed to analyze the cell-mediated immune responses in viral infection. Generally, the chromium $\left({ }^{51} \mathrm{Cr}\right)$-release assay has been the most popularly used for its high sensitivity and antigenic specificity. However, this assay requires environmental and special techniques due to the use of short-lived radioactive materials. Moreover, it is difficult to handle virus-infected samples such as those with human immunodeficiency virus (HIV). Recently, several alternative non-radioactive assays have been developed to avoid radioisotope use. In particular, highly

Address correspondence to: Yohko Nakagawa

Department of Microbiology and Immunology, Nippon Medical School, 1-1-5 Sendagi, Bunkyo-ku, Tokyo 113-8602, Japan

Tel: +81-3-3822-2131 (ext. 5367), Fax: +81-3-3827-3381

E-mail: tanuki@nms.ac.jp (Y. Nakagawa) sensitive flow cytometric assays using various fluorescent-associated dyes such as c'FDA (10), DiO (3), FITC (7), Calcein-AM (11), PKH26 and PKH2 (4) have been reported. Although some of these assays can detect the cytotoxic activities of CTLs and concurrently examine the characterization of cells involved in the cytolytic reaction, they are not suitable to quickly evaluate many samples because of the complicated two-color dot blot analysis on an FACScan analyzer.

Recently, method to determine antigen-specific cytotoxic activities of CTLs in vivo have been established by using fluorescent-based dye 5- (and 6-) carboxyfluorescein diacetate succinimydyl ester (CFSE) $(1,2)$. In this assay, antigen-specific cytotoxic activities in cells from various murine lymphoid tissues can be detected by flow cytometry after injection of control and sensitive target cells labeled with different concentrations of CFSE.

\footnotetext{
*Both have the same contribution to this work.
} 
Therefore, we applied this dye to an in vitro assay system and established a simple, highly sensitive flow cytometric assay to detect CTL activity (FACSCTL assay). In our assay, specific activities of CTLs could be detected as a reduction in sensitive target cell numbers on single-color histogram plot analysis.

Because our assay uses only a stable non-radioactive reagent, CFSE, this assay is safe, inexpensive and extremely easy. The results obtained in this study suggest that this simple assay could be an acceptable alternative to the classical ${ }^{51} \mathrm{Cr}$-release assay.

\section{MATERIALS AND METHODS}

Mice. Female BALB/c $\left(\mathrm{H}-2^{\mathrm{d}}\right)$ mice were purchased from Charles-River Japan Inc. (Tokyo, Japan). Transgenic mice (Tg-RT1) expressing TCR $\alpha$ and $\beta$ chains (V $\alpha$ 42H11 and V $\beta 8.1$ ) genes of a murine CTL clone RT-1 (13) specific for P18-I10 restricted by a $\mathrm{D}^{\mathrm{d}}$ class I MHC molecule $(12,16)$ and carrying the genetic background of BALB/c were established and bred in our colony (17). All mice used in this study were maintained in a specific pathogen-free microisolator environment. All experiments were performed according to the guidelines of the NIH Guide for the Care and Use of Laboratory Animals.

Recombinant vaccinia virus. vSC-25 (recombinant vaccinia virus expressing the HIV-1, IIIB gp160 envelope glycoprotein) was described previously (12) and was used to infect mice to induce envelope-specific CTL. For infection experiments, mice were intraperitoneally inoculated with $1 \times 10^{7} \mathrm{PFU} /$ mouse of vSC-25.

Synthetic peptides and reagents. Peptides used in this study were all purchased from Takara Bio Co. (Tokyo, Japan). Peptide P18IIIB (aa: RIQRGPGRAF VTIGK) is the immunodominant CTL epitope presented by the murine class I MHC molecule $\mathrm{H}-2 \mathrm{D}^{\mathrm{d}}$ in the V3 loop of the HIV-1 gp160 glycoprotein found in strain IIIB (12) and peptide P18-I10 (aa: RGPGRAFVTI) is the minimal active 10-mer peptide within P18IIIB (16). Control peptide P18MN (aa: RIHIGPGRAFYTTKN), P18RF (aa: SITKGPG RVIYATGQ) and P18MN substituted peptides (See Fig. 3A) were also used.

A Cell Trace ${ }^{\mathrm{TM}}$ CFSE cell proliferation Kit [Flourescein-based dye 5- (and 6-) carboxyfluorescein diacetate succinimydyl ester; CFSE] was purchased from Life Technology Corporation (Carlsbad, CA, USA) and dissolved in dimethylsulfoxide at a concentra- tion of $5 \mathrm{mM}$ according to the manufacturer's instructions. This stock solution was kept at $-20^{\circ} \mathrm{C}$ until experiments. Calibration beads were purchased from Polysciences Inc. (Warrington, PA, USA).

Cell lines. P815 cells (a DBA/2-derived mastocytoma cell line; $\mathrm{H}-2^{\mathrm{d}}$ ) were used as target cells. BALB/ c.3T3 $\left(\mathrm{H}-2^{\mathrm{d}}\right)$ fibroblast transfectants expressing HIV-1 gp160 of the IIIB isolate (15-12 cells) and control transfectants with only the selectable marker genes (Neo) were described previously (12) and were also used as target cells. These lines were used with more than ninety percent viability. The CTL line (LINE-IIIB) specific for the HIV-1 envelope protein of the IIIB-strain has been described previously $(12,13)$. This CTL line was restricted by the $\mathrm{D}^{\mathrm{d}}$ class I MHC molecule and is specific for an immunodominant epitope of HIV-1 gp160, P18IIIB (12).

Cytotoxicity assay using the CFSE fluorescent-based dye. On the day of the assay, peptide P18-I10-pulsed P815 cells or unpulsed control P815 target cells were labeled with CFSE as follows: Cells were suspended in phosphate-buffered saline (PBS) containing $1 \% \mathrm{FCS}$ and adjusted to $1 \times 10^{6} / \mathrm{mL}$. For sensitive targets, $0.5 \mu \mathrm{L}$ of CFSE stock solution $(5 \mathrm{mM})$ was added to $1 \mathrm{~mL}$ of cell suspension and incubated for $4 \mathrm{~min}$ at room temperature under shading. For control targets, $0.5 \mu \mathrm{L}$ of diluted CFSE solution $(100 \mu \mathrm{M})$ was used for labeling. After incubation, $9 \mathrm{~mL}$ of PBS containing 5\% FCS (FCS-PBS) was added to stop the labeling reaction. Then, cells were washed once with FCS-PBS and resuspended with complete T cell medium (CTM) (12). Effector cells were washed once and resuspended in CTM to a final concentration of $1.25-5 \times 10^{6} / \mathrm{mL}$. For sensitive reactions, $5 \times 10^{4}$ of peptide P18-I10-pulsed P815 target cells and various numbers of effector cells were added to round-bottom polystyrene tubes (Falcon, Becton Dickinson Labware, Franklin Lakes, $\mathrm{NJ}$ ) at a final volume of $500 \mu \mathrm{L}$ to yield various effector/target $(\mathrm{E} / \mathrm{T})$ ratios. Control tubes containing 5 $\times 10^{4}$ of unpulsed target cells and the same effector cells were also separately prepared. Tubes were gently mixed, centrifuged at $200 \times g$ for $1 \mathrm{~min}$, and then incubated at $37^{\circ} \mathrm{C}$ for $4 \mathrm{~h}$. After incubation, sensitive target cells were mixed with control target cells in one tube with PBS containing 1\% FCS and $0.1 \%$ sodium azide (FACS buffer) at the same E/T ratio and mixed cells were washed once with FACS buffer and resuspended with 4\% paraformaldehyde (PFA; Sigma)-containing PBS (PFA-PBS). 
Flow cytometric acquisition and calculation. Acquisition was performed using a FACScan analyzer (Beckton Dickinson Immunocheminal Systems, Mountain View, CA) with Cell Quest software (Beckton Dickinson). A detailed description of the sample acquisition and analysis is shown in results. All samples were assayed in duplicate and the mean percentage of specific lysis was calculated as follows: $\%$ specific lysis $=[$ (number of sensitive target cells in control sample - number of sensitive target cells in test sample / number of sensitive target cells in control sample) $] \times 100$. In this formula, number of cells refers to the event count on Cell Quest software. Control sample indicates target cells incubated without added effector cells and test sample shows target cells incubated with added effector cells.

${ }^{51} \mathrm{Cr}$ release assay. A ${ }^{51} \mathrm{Cr}$-release assay was performed as previously described (12).

\section{RESULTS}

Detection of antigen-specific cytotoxicity of CTLs by FACS-CTL assay

We have already established a $\mathrm{CD} 8^{+}, \mathrm{H}-2 \mathrm{D}^{\mathrm{d}}$ class I MHC molecule-restricted, murine cytotoxic $\mathrm{T}$ lymphocyte (CTL) line, LINE-IIIB, specific for the envelope glycoprotein 160 (gp160) of the IIIB strain of HIV-1 (12). Furthermore, we identified an immunodominant epitope within gp160 as a 15-mer peptide, P18IIIB (aa: RIQRGPGRAFVTIGK), as well as the minimal active 10-mer peptide, P18-I10 (aa: RGPGRAFVTI) within P18IIIB (16). By using this CTL line and P815 cells as effector and target cells, respectively, we examined the basic conditions for our FACS-CTL assay. Peptide pulsed-P815 cells were labeled with a high concentration of CFSE and used for sensitive target cells, while unpulsed P815 cells were labeled with a low concentration of CFSE and were used as control target cells. After labeling with CFSE reagent, these target cells were separately incubated with various numbers of LINE-IIIB for $4 \mathrm{~h}$ at $37^{\circ} \mathrm{C}$. After incubation, sensitive targets were mixed with control targets in one tube with FACS buffer at the same E/T ratio. Mixed target cells were washed once with FACS buffer and resuspended with PFA-PBS to stop any additional reaction. In acquisition of the cells by the FACScan analyzer, five thousand cells which were contained in the gate consisted of P815 cells (region 1; R1) and two CFSE peaks on a histogram plot (region 2; R2) were acquired (see Fig. 1).

First, to see whether antigenic specificities could
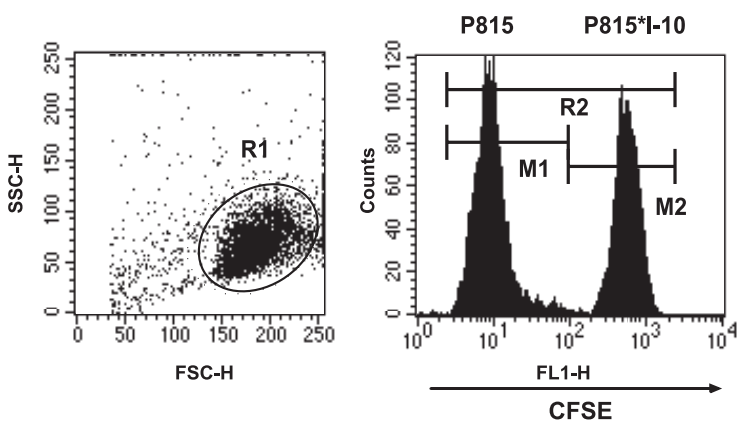

Fig. 1 Acquisition on flow cytometry. Left: Target cells containing $2.5 \mu \mathrm{M}$ of CFSE-labeled, peptide P18-I10-pulsed P815 cells and $50 \mathrm{nM}$ of CFSE-labeled, unpulsed P815 cells appeared in a gated region (R1) on dot blot analysis by FACScan analyzer. Right: Histogram profile of the cells in the gated region (R1) is shown. For acquisition, $5 \times 10^{3}$ cells which were contained in both regions, $\mathrm{R} 1$ and $\mathrm{R} 2$, were collected.

be detected by FACS-CTL assay, cytotoxic activities of LINE-IIIB against an epitope peptide, P18-I10pulsed P815 cells, or a control peptide, P18RFpulsed P815 cells, were determined, respectively. As shown in Fig. 2, after $4 \mathrm{~h}$ incubation the number of P18-I10-pulsed P815 cells was significantly reduced in proportion to the number of added effector cells. In contrast, when LINE-IIIB was incubated with P18RF-pulsed or unpulsed P815 cells, no reduction in fluorescence was observed. These results clearly indicated that antigen-specific cytotoxic activities could be detected by this FACS-CTL assay.

In previous studies, we demonstrated that the amino acid at position 325 plays a critical role in the specific recognition of LINE-IIIB to epitope peptide P18IIIB (14, 15). Although this CTL line could not recognize peptide $\mathrm{P} 18 \mathrm{MN}$, the same immunodominant site from another strain of HIV-1, it could cross-recognize the substituted peptide P18MN(Y-I) at which position 325(Y) was replaced by isoleucine (I) (15). As shown in Fig. 3, fluorescence of target cells sensitized with P18IIIB and P18MN(Y-I) was significantly reduced after incubation with LINE-IIIB. In contrast, the number of target cells pulsed with peptide P18MN or P18MN(Y-E), which is not recognized by LINE-IIIB, was unchanged. These results indicate that changes in cytotoxic activity due to single amino acid substitution within an epitope peptide could be detected by the FACS-CTL assay.

Usage of adherent cells as target cells on FACSCTL assay

In some experiments, we used adherent cells such as BALB/c.3T3-derived fibroblast cells as target 
(A)

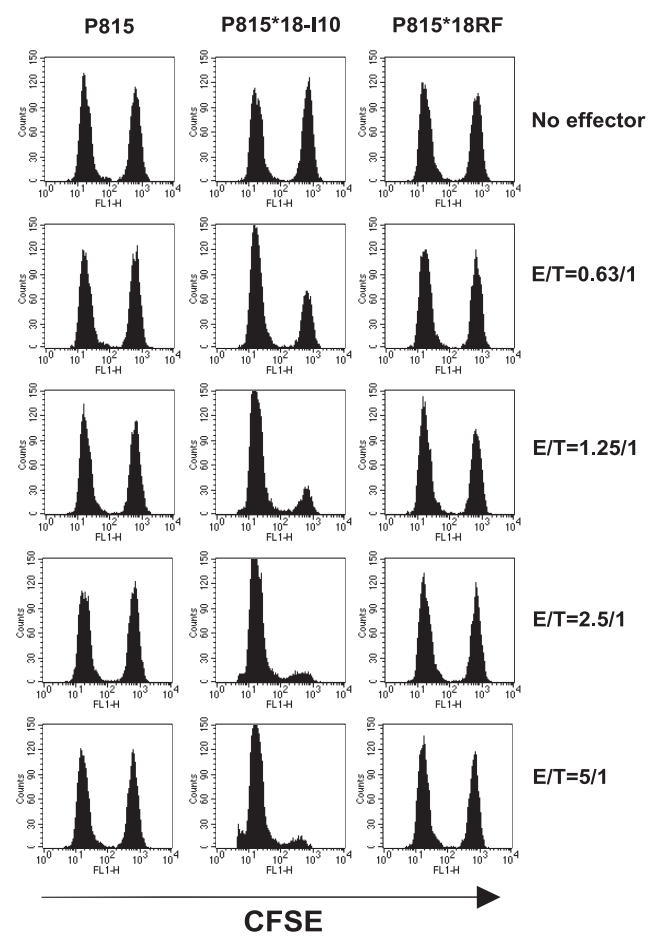

(B)

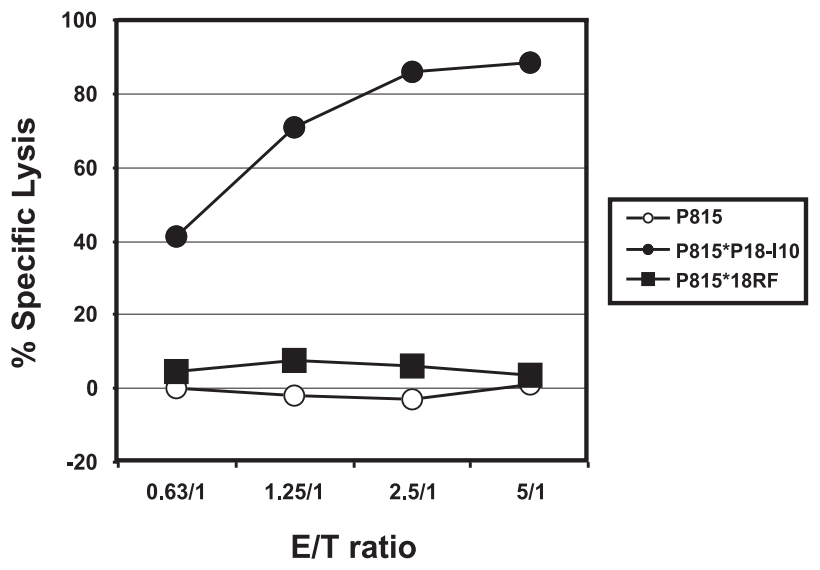

Fig. 2 Detection of antigen-specific cytotoxicity by FACSCTL assay. (A) For sensitive targets, epitope peptide P18I10-pulsed, control peptide P18RF-pulsed or unpulsed P815 cells were labeled with $2.5 \mu \mathrm{M}$ of CFSE, respectively. For control targets for acquisition, unpulsed P815 cells were labeled with $50 \mathrm{nM}$ of CFSE. Each labeled target cells $(5 \times$ $10^{4}$ ) were co-incubated with effector CTLs (LINE-IIIB) at various effector/target $(\mathrm{E} / \mathrm{T})$ ratios in $5 \mathrm{~mL}$ polystyreneround bottom tubes in a $500 \mu \mathrm{L}$ final volume for $4 \mathrm{~h}$ at $37^{\circ} \mathrm{C}$. After incubation, sensitive targets were mixed with control targets in one tube at the same $E / T$ ratio and mixed cells were washed once with FACS buffer and resuspended with PFA-PBS. Histogram profiles of each sample are shown. (B) Percent specific lysis calculated by a formula is shown (see Materials and Methods).
(A)

\begin{tabular}{lc}
\hline \multicolumn{1}{c}{ Peptides } & Sequences \\
\hline 18 IIIB & RIQRGPGRAFVTIGK \\
$18 \mathrm{MN}$ & RIHIGPGRAFYTTKN \\
$18 \mathrm{MN}(\mathrm{Y}-\mathrm{I})$ & RIHIGPGRAFITTKN \\
$18 \mathrm{MN}(\mathrm{Y}-\mathrm{E})$ & RIHIGPGRAFETTKN \\
\hline
\end{tabular}

(B)

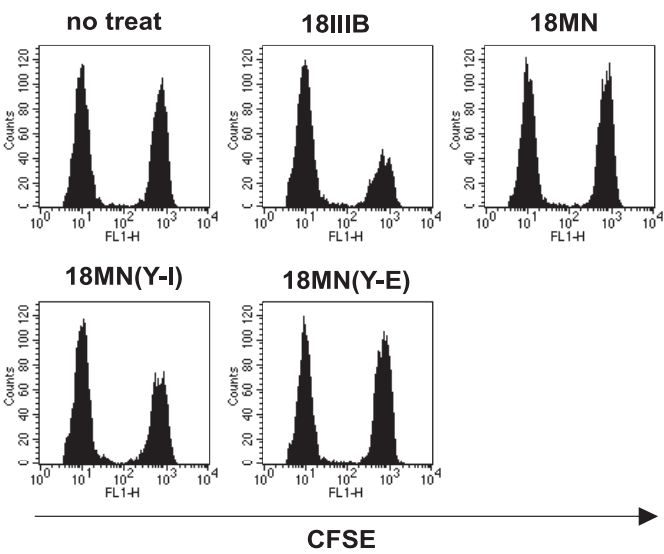

(C)

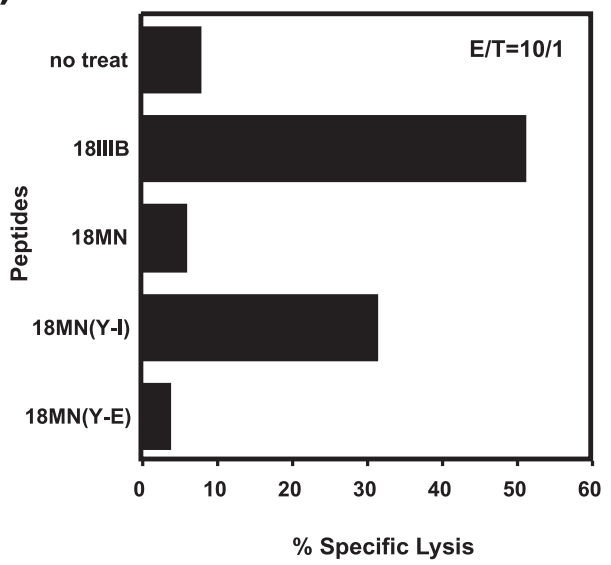

Fig. 3 Detection of fine antigenic specificity by FACS-CTL assay. For sensitive targets, epitope peptide P18IIIB-pulsed, control peptide P18MN-pulsed or P18MN substituted peptides-pulsed P815 cells were labeled with $2.5 \mu \mathrm{M}$ of CFSE, respectively. For control targets for acquisition, unpulsed P815 cells were labeled with $50 \mathrm{nM}$ of CFSE. Assays were done the same as in Fig. 2. Histogram profile of each sample and percent specific lysis calculated by a formula $(E / T$ ratio $=10 / 1$ ) are shown. 
cells in the ${ }^{51} \mathrm{Cr}$-release assay. However, when CTLs were incubated with adherent target cells for $4 \mathrm{~h}$ in the FACS-CTL assay, most target cells adhered to inside of the assay tubes, and so we could not collect the target cells after incubation. Therefore, we tried to collect target cells by treatment with high concentrations of EDTA and trypsin solutions. After $4 \mathrm{~h}$ incubation, a $100 \mu \mathrm{L}$ aliquot of $2 \%$ EDTA solution and a $100 \mu \mathrm{L}$ aliquot of $5 \%$ trypsin solution were added to each assay tube and further incubated for $10 \mathrm{~min}$ at $37^{\circ} \mathrm{C}$. After incubation, cells were stirred for $5 \mathrm{~s}$ and then sensitive and control target cells were mixed with FACS buffer in one tube for FACS analysis. As shown in Fig. 4, most adherent target cells such as 15-12 cells and Neo were harvested with this treatment and the cytotoxic activities of LINE-IIIB against these adherent target cells could be detected by the FACS-CTL assay.

The accuracy of FACS-CTL assay and comparison with standard ${ }^{5 I} \mathrm{Cr}$-release assay

To confirm the accuracy of our FACS-CTL assay, we next assessed the same samples in quadruplicate

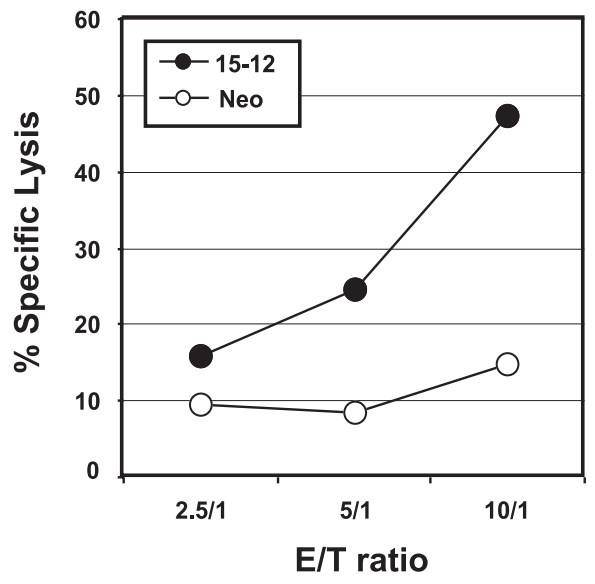

Fig. 4 Cytotoxic activities of LINE-IIIB were determined by FACS-CTL assay using HIV-1 gp160 gene-transfected BALB/ c.3T3 cells (15-12) and control BALB/c.3T3 cells (Neo) as target cells. For sensitive targets, 15-12 or Neo was labeled with $2.5 \mu \mathrm{M}$ of CFSE, respectively, and for control targets, Neo was labeled with $50 \mathrm{nM}$ of CFSE. Each sample of labeled target cells was incubated with LINE-IIIB at various $\mathrm{E} / \mathrm{T}$ ratios for $4 \mathrm{~h}$ at $37^{\circ} \mathrm{C}$. After incubation, $100 \mu \mathrm{L}$ of $2 \%$ EDTA solution and $100 \mu \mathrm{L}$ of $5 \%$ trypsin solution were added to each sample and further incubated for $10 \mathrm{~min}$ at $37^{\circ} \mathrm{C}$. After incubation, cells were stirred by a voltex mixer three times. Sensitive and control target cells were mixed in one tube at each $E / T$ ratio and mixed cells were washed once with FACS buffer and resuspended with PFA-PBS for FACS analysis. Open symbols, both sensitive and control targets were $\mathrm{Neo}$; closed symbols, the sensitive target was 15-12 and control target was Neo. at each E/T ratio. The results clearly indicated that the FACS-CTL assay was very accurate because little standard error was detected at all $\mathrm{E} / \mathrm{T}$ ratios (Fig. 5A).

The ${ }^{51} \mathrm{Cr}$-release assay has been widely used to examine the cytotoxic activities of CTLs against various virus-infected cells or tumor cells. Therefore, next we examined the cytotoxicities of LINEIIIB using the FACS-CTL assay and compared the results with those obtained by a ${ }^{51} \mathrm{Cr}$-release assay. A direct comparison of the two assays was performed concurrently using identical effectors, target cells and $\mathrm{E} / \mathrm{T}$ ratios. In these experiments, two LINE-IIIB cells with different activities were used

\section{(A)}

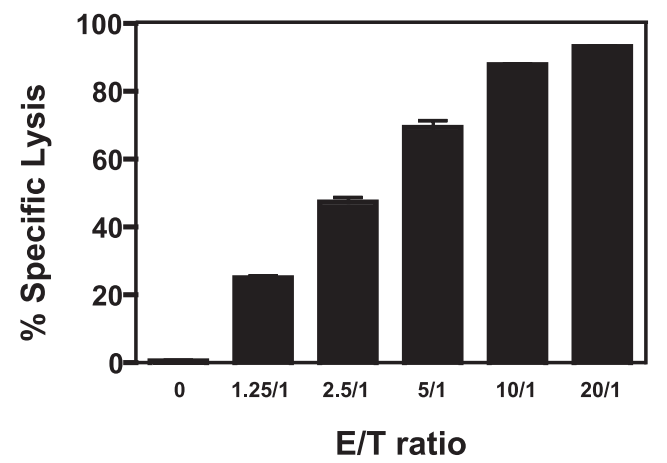

(B)

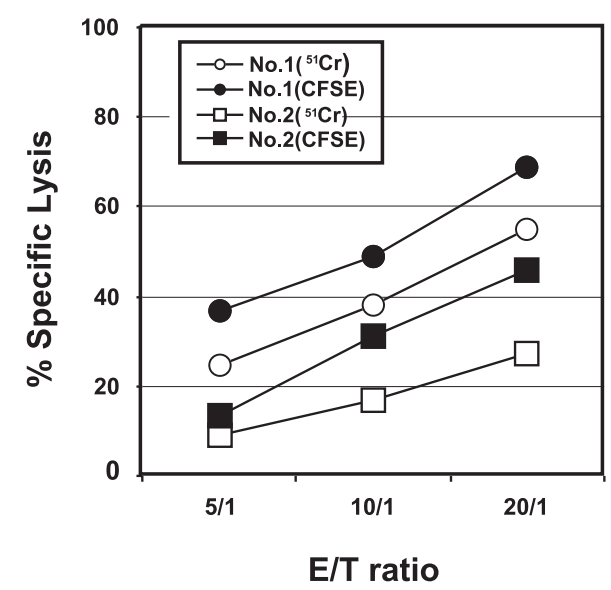

Fig. 5 The accuracy of FACS-CTL assay and comparison with standard ${ }^{51} \mathrm{Cr}$-release assay. (A) The same samples were determined in quadruplicate at each $E / T$ ratios on FACS-CTL assay. LINE-IIIB cells and P18-I10-pulsed and unpulsed P815 cells were used as effector and target cells, respectively. (B) Cytotoxic activities of two LINE-IIIB cells with different activity were determined by ${ }^{51} \mathrm{Cr}$-release assay (open symbols) and FACS-CTL assay (closed symbols) using P18-I10-pulsed and unpulsed P815 cells as target cells. 
as effector cells. As shown in Fig. 5 (B), the percent specific lysis obtained by our assay was in close agreement with experimental data obtained from a ${ }^{51} \mathrm{Cr}$-release assay.

Furthermore, we examined the stability of prepared samples. After cells were resuspended with PFA-PBS, acquisition was done just after preparation and also two and three days after preparation. As shown in Table 1, almost the same percent lysis was obtained at these three acquisition times. These results indicate that we could stock prepared samples under shading from light for a few days after preparation.

Ex vivo CTL assay using the FACS-CTL assay

Next, an ex vivo CTL assay was done with the same protocol using spleen cells derived from virusinfected mice. Spleen cells were prepared from vSC25 -infected transgenic mice or normal BALB/c mice and used as effector cells for assays. As shown in Fig. 6, antigen-specific cytotoxic activities in spleen cells from infected mice could be determined by our assay with high sensitivity.

Determination of CTL activity against tumor cells It is important to analyze the cytotoxic activities of CTLs against tumor cells. However, when tumor cells are used as sensitive targets, we often can not provide control targets for assays. Therefore, we tried to use calibration beads as a control for this assay. Because the fluorescent intensity of the calibration beads was strong, 15-12 cells were labeled with a low concentration of CFSE (final concentration at $50 \mathrm{nM}$ ) and incubated with various numbers of LINE-IIIB for $4 \mathrm{~h}$ at $37^{\circ} \mathrm{C}$. After incubation, a $10 \mu \mathrm{L}$ aliquot of diluted calibration beads was added to each sample and five thousand cells, which were contained in the gate consisting of region 1 (15-12 cells and beads) and two fluorescent peaks on a histogram plot (region 2; R2), were acquired. As

Table 1 Stability of prepared FACS-CTL assay samples

\begin{tabular}{rccc}
\hline E/T ratio & \multicolumn{3}{c}{ \% Specific Lysis } \\
\hline & Day 0 & Day 2 & Day 3 \\
\cline { 2 - 4 } $5 / 1$ & 10.9 & 11.0 & 17.0 \\
$10 / 1$ & 29.4 & 31.7 & 33.6 \\
$20 / 1$ & 46.5 & 52.5 & 52.3 \\
\hline
\end{tabular}

$4 \%$ paraformaldehyde-fixed samples were assessed just after preparation (day 0), and two days (day 2) and three days (day 3) after preparation by a FACScan analyzer. LINE-IIIB cells were used as effector cells. Percent specific lysis against P18-I10pulsed P815 cells is shown. shown in Fig. 7, even when calibration beads were used as a control for the assay, the cytotoxic activities of LINE-IIIB against 15-12 cells could be detected and the results obtained from the assay were in close agreement with the data obtained by using $\mathrm{Neo}$ as a control.

\section{DISCUSSION}

In this paper, we reported a rapid and simple assay

(A)
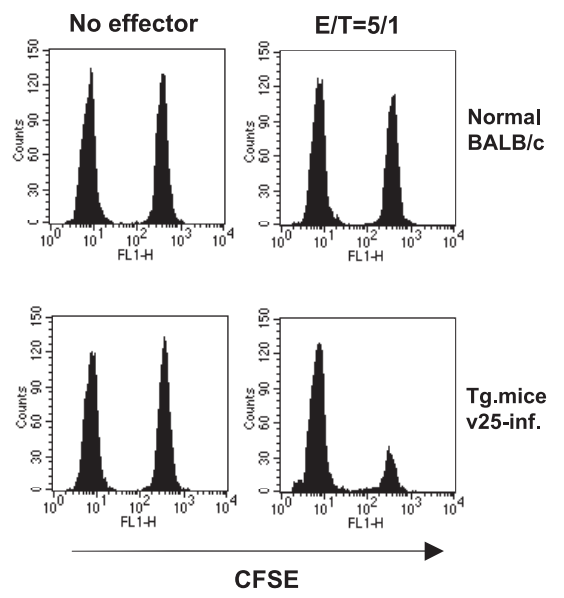

(B)

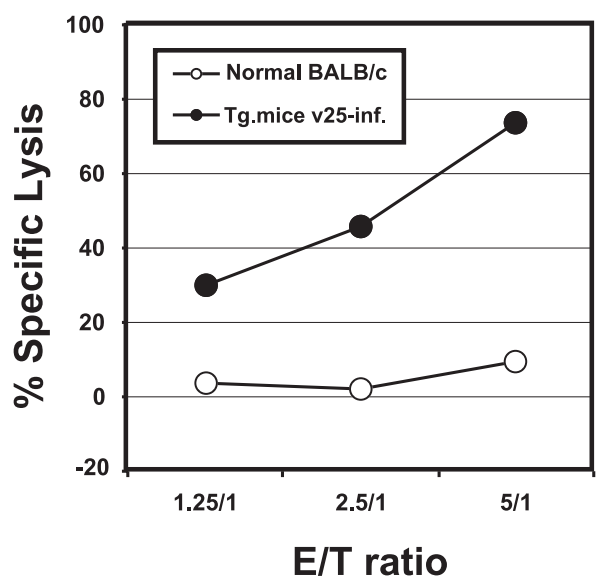

Fig. 6 Ex vivo CTL assay using the FACS-CTL assay. TgRT1 mice were intraperitoneally inoculated with $1 \times 10^{7}$ PFU of vSC-25. Seven days after inoculation, spleen cells were prepared from vSC-25-inoculated Tg-RT1 mice and from control normal BALB/c mice. Cytotoxic activities of these spleen cells were determined by FACS-CTL assay using peptide P18-I10-pulsed and unpulsed P815 cells as sensitive and control target cells, respectively. Histogram profiles $(A)$ and percent specific lysis calculated by a formula $(B)$ are shown. 
to assess antigen-specific cytotoxic activities of CTLs in vitro using single fluorescent-dye CFSE. Recently, several flow cytometric assays to detect CTL activity using CFSE have been reported by others $(5,6,8)$, but are still not widely used. From some of these reports, we can get more detailed information such as phenotyping of target cells or effector cell subsets other than specific cytotoxicity. However, these assays may not be easy to perform

\section{(A)}

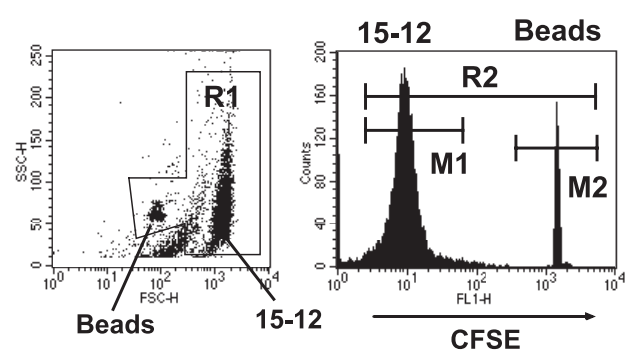

(B)

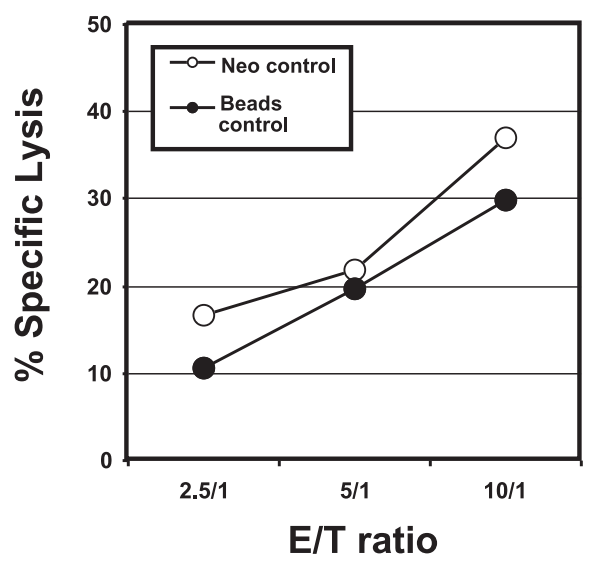

Fig. 7 FACS-CTL assay using fluorescent calibration beads as a control. (A) Gated region (R1) containing $50 \mathrm{nM}$ of CFSE-labeled 15-12 cells and calibration beads on dot blot analysis and histogram profile of the cells in the gated region (R1) by FACScan analyzer are shown. For acquisition, $5 \times 10^{3}$ cells which were contained in both regions $\mathrm{R} 1$ and R2 were collected. (B) Cytotoxic activities of LINE-IIIB were determined by FACS-CTL assay using fluorescent calibration beads and 15-12 cells. 15-12 cells were labeled with $50 \mathrm{nM}$ of CFSE and incubated with LINE-IIIB at various $E / T$ ratios for $4 \mathrm{~h}$ at $37^{\circ} \mathrm{C}$. After incubation, cells were treated with EDTA and trypsin solutions, washed with FACS buffer and resuspended with PFA-PBS. Ten $\mu \mathrm{L}$ aliquots of diluted calibration beads were added to each sample just before acquisition (closed symbols). For control experiments, cytotoxic activities of the same LINE-IIIB were determined using $2.5 \mu \mathrm{M}$ of CFSE-labeled 15-12 cells and $50 \mathrm{nM}$ of CFSE-labeled Neo were used as sensitive and control target cells, respectively (open symbols). because they require several steps and complicated two-color dot blot analysis. Although it was possible to detect only the cytotoxic activity in our FACSCTL assay, this assay is extremely simple; thus, it is suitable to quickly determine many samples similar to the ${ }^{51} \mathrm{Cr}$-release assay. Moreover, with some improvements such as treatment with excess EDTA/ trypsin reagents or using fluorescent calibration beads as a control, adherent cells and tumor cells also could be used as target cells. Although propidium iodide was not added to the samples to detect dead cells after incubation, it was not a problem in the analysis by our system because we always used target cells with high viability. Results obtained from this study suggest that the FACS-CTL assay is an acceptable alternative to the classical ${ }^{51} \mathrm{Cr}$-release assay.

Furthermore, our FACS-CTL assay has some advantages over to the ${ }^{51} \mathrm{Cr}$-release assay. First, in our assay, prepared samples are fixed with PFA, so samples with inherent risk such as virally infected cells can also be determined. Second, non-activating cells may be used as target cells. In the ${ }^{51} \mathrm{Cr}$-release assay, resting cells or non-dividing cells such as murine naïve splenocytes (our unpublished observation) or neuronal cells do not easily incorporate ${ }^{51} \mathrm{Cr}$ and so it is difficult to use these cells as target cells. In contrast, these cells are labeled with CFSE the same as activating cells $(1,2,9)$ because the CFSE reagent passively diffuses into these cells. We could also use these cells as targets in the FACS-CTL assay. Third, since the fluorescence of prepared samples is stable for as long as three days, it is possible to analyze samples with a FACScan analyzer even if they are stocked or transported from another area.

The FACS-CTL assay described in this study will allow precise quantitative measurement of cell-mediated cytotoxity and analysis of the fine mechanism of CTL function in the virological and immunological fields, including cancer research, and diverse situations in which laboratory restrictions exist concerning the use of radioisotopes.

\section{Acknowledgements}

This work was supported in part by grants from the Ministry of Education, Science, Sport, and Culture, from the Ministry of Health and Labor and Welfare, Japan, and from the Japanese Health Sciences Foundation, and by the Promotion and Mutual Aid Corporation for Private School of Japan. 


\section{REFERENCES}

1. Barber DL, Wherry EJ and Ahmed R (2003) Cutting edge: rapid in vivo killing by memory CD8 T cells. J Immunol 171, 27-31.

2. Byers AM, Kemball CC, Moser JM and Lukacher AE (2003) Cutting edge: rapid in vivo CTL activity by polyoma virusspecific effector and memory $\mathrm{CD}^{+} \mathrm{T}$ cells. J Immunol $\mathbf{1 7 1}$, 17-21.

3. Chang L, Gusewitch GA, Chritton DB, Folz JC, Lebeck LK and Nehlsen-Cannarella SL (1993) Rapid flow cytometric assay for the assessment of natural killer cell activity. $J$ Іттиnol Methods 166, 45-54.

4. Flieger D, Gruber R, Schlimok G, Reiter C, Pantel K and Riethmuller G (1995) A novel non-radioactive cellular cytotoxicity test based on the differential assessment of living and killed target and effector cells. J Immunol Methods 180, $1-13$.

5. Godoy-Ramirez K, Makitalo B, Thorstensson R, Sandstrom E, Biberfeld $G$ and Gaines H (2005) A novel assay for assessment of HIV-specific cytotoxicity by multiparameter flow cytometry. Cytometry A 68, 71-80.

6. Jedema I, van der Werff NM, Barge RM, Willemze R and Falkenburg JH (2004) New CFSE-based assay to determine susceptibility to lysis by cytotoxic $\mathrm{T}$ cells of leukemic precursor cells within a heterogeneous target cell population. Blood 103, 2677-2682.

7. Karawajew L, Jung G, Wolf H, Micheel B and Ganzel K (1994) A flow cytometric long-term cytotoxicity assay. J Immunol Methods 177, 119-130.

8. Lecoeur H, Fevrier M, Garcia S, Riviere Y and Gougeon ML (2001) A novel flow cytometric assay for quantitation and multiparametric characterization of cell-mediated cytotoxicity. J Immunol Methods 253, 177-187.

9. Li X, Dancausse H, Grijalva I, Oliveira M and Levi AD (2003) Labeling Schwann cells with CFSE-an in vitro and in vivo study. J Neurosci Methods 125, 83-91.

10. McGinnes K, Chapman G, Marks R and Penny R (1986) A fluorescence NK assay using flow cytometry. J Immunol
Methods 86, 7-15.

11. Papadopoulos NG, Dedoussis GV, Spanakos G, Gritzapis AD, Baxevanis CN and Papamichail M (1994) An improved fluorescence assay for the determination of lymphocytemediated cytotoxicity using flow cytometry. J Immunol Methods 177, 101-111.

12. Takahashi H, Cohen J, Hosmalin A, Cease KB, Houghten R, Cornette JL, DeLisi C, Moss B, Germain RN and Berzofsky JA (1988) An immunodominant epitope of the human immunodeficiency virus envelope glycoprotein gp160 recognized by class I major histocompatibility complex molecule-restricted murine cytotoxic T lymphocytes. Proc Natl Acad Sci U S A 85, 3105-3109.

13. Takahashi H, Houghten R, Putney SD, Margulies DH, Moss B, Germain RN and Berzofsky JA (1989) Structural requirements for class I MHC molecule-mediated antigen presentation and cytotoxic $\mathrm{T}$ cell recognition of an immunodominant determinant of the human immunodeficiency virus envelope protein. J Exp Med 170, 2023-2035.

14. Takahashi H, Merli S, Putney SD, Houghten R, Moss B, Germain RN and Berzofsky JA (1989) A single amino acid interchange yields reciprocal CTL specificities for HIV-1 gp160. Science 246, 118-121.

15. Takahashi H, Nakagawa $Y$, Pendleton CD, Houghten RA, Yokomuro K, Germain RN and Berzofsky JA (1992) Induction of broadly cross-reactive cytotoxic $\mathrm{T}$ cells recognizing an HIV-1 envelope determinant. Science 255, 333-336.

16. Takeshita T, Takahashi H, Kozlowski S, Ahlers JD, Pendleton CD, Moore RL, Nakagawa Y, Yokomuro K, Fox BS, Margulies DH and Berzofsky JA (1995) Molecular analysis of the same HIV peptide functionally binding to both a class I and a class II MHC molecule. J Immunol 154, 1973-1986.

17. Yokosuka T, Takase K, Suzuki M, Nakagawa Y, Taki S, Takahashi H, Fujisawa T, Arase H and Saito T (2002) Predominant role of $\mathrm{T}$ cell receptor (TCR)-alpha chain in forming preimmune TCR repertoire revealed by clonal TCR reconstitution system. $J$ Exp Med 195, 991-1001. 